\title{
ENDOSCOPY AND AUTOPSY FOLLOW-UP OF BIODEGRADABLE OESOPHAGEAL ANASTOMOSES IN DOGS
}

\author{
T. KOVÁCs ${ }^{1 *}$, T. NÉMETH ${ }^{2}$, Zs. OROSZ ${ }^{3}$ and I. KÖVES ${ }^{1}$ \\ ${ }^{1}$ Department of General and Thoracic Surgery, National Institute of Oncology, \\ H-1122 Budapest, Ráth Gy. u. 7-9, Hungary; ${ }^{2}$ Department and Clinic of Surgery and \\ Ophthalmology, 'Szent István' University, Faculty of Veterinary Science, Budapest, \\ Hungary; ${ }^{3}$ Department of Pathology, National Institute of Oncology, Budapest, Hungary
}

(Received May 25, 2001; accepted October 5, 2001)

The Biofragmentable Anastomosis Ring (BAR) is a mechanical device composed of absorbable material and creates an inverting, atraumatic compressive anastomosis with no foreign material at the anastomotic site after healing. The aim of the present experimental study was to assess the safety of oesophagoscopy in early days after oesophageal anastomoses performed with the BAR and to follow-up the healing of BAR anastomoses by in vivo endoscopy and autopsy examination. Thirty mongrel dogs divided into subgroups according to the timepoints of endoscopy and autopsy (4th, 7th, 14th, 28th day) were used. There was no significant difference in the healing of anastomoses performed under or above the tracheal bifurcation. Pleural adhesions helped to cover and seal small subclinical leaks. The mortality was $13.3 \%$ (4 dogs) and the overall leakage rate $14.3 \%$. We looked for bleeding, haematoma, erosion, ulceration and granulation tissue in the anastomosis. Due to the high mechanical strength of these anastomoses, oesophagoscopy was a safe, easy and feasible method for follow-up BAR intrathoracic anastomoses, with no significant difference between the number of lesions found with endoscopy as compared to the autopsy data. The overall sensitivity of oesophagoscopy to discover mucosal lesions was $73.1 \%$. Endoscopy had no complications, therefore it is a useful method of follow-up and may help predict the normal or compromised healing of oesophageal anastomoses.

Key words: Endoscopy, autopsy follow-up, biofragmentable oesophageal anastomosis, dog

The Biofragmentable Anastomosis Ring (BAR) is a mechanical anastomotic device made of absorbable material (polyglycolic acid $87.5 \%$ and barium sulphate $12.5 \%$ ), and is used to create anastomoses on the gastrointestinal (GI) tract from the stomach to the middle third of the rectum (Thiede et al., 1998). The BAR (VALTRAC ${ }^{\circledR}$ produced by Davis \& Geck) creates an inverting anastomosis by atraumatic apposition of the bowel ends. It is eliminated from the site of the anastomosis with the stools by hydrolysis and fragmentation. There re-

\footnotetext{
*Corresponding author; E-mail:kovacsti@oncol.hu; Fax: +36 (1) 224-8620
} 
mains no foreign material in the bowel wall, therefore strictures are extremely rare. The method is simple, quick and safe, it has a standard technique all over the GI tract which is easy to learn (Hardy et al., 1987; Kovács et al., 1996). The BAR has been already used to create cervical oesophageal anastomoses in dogs (Dietz, 1994; Dietz et al., 1995), but authors reported a high leakage rate explained by the compression ischaemic lesions produced by the tracheal cartilages in contact with the oesophageal wall and ring. There are no experimental reports regarding the use of the BAR for intrathoracic oesophageal anastomoses.

In the present experimental work we studied the healing of oesophageal anastomoses performed with the BAR on the intrathoracic oesophagus of dogs under and above the tracheal bifurcation (Kovács et al., 1998).

Usually endoscopy is not recommended in the early postoperative days after digestive anastomoses, because the examination may damage the fresh anastomosis. Oesophagoscopy would be useful to detect bleeding, haematoma, ischaemic lesions and strictures in the area of the anastomosis (Bundy et al., 1992). The aim of the study was to assess whether it is safe to do oesophagoscopy after oesophageal anastomoses performed with the BAR and to follow-up the healing of oesophageal BAR anastomoses by in vivo endoscopy and autopsy examination (macroscopic gross examination).

\section{Material and methods}

The study was carried out in accordance with the 'Guide for the Care and Use of Laboratory Animals' of the Faculty of Veterinary Science, Budapest, Hungary.

The BAR is a mechanical device with two mushroom-shaped headpieces unified by a central interdigitating segment (Fig. 1).

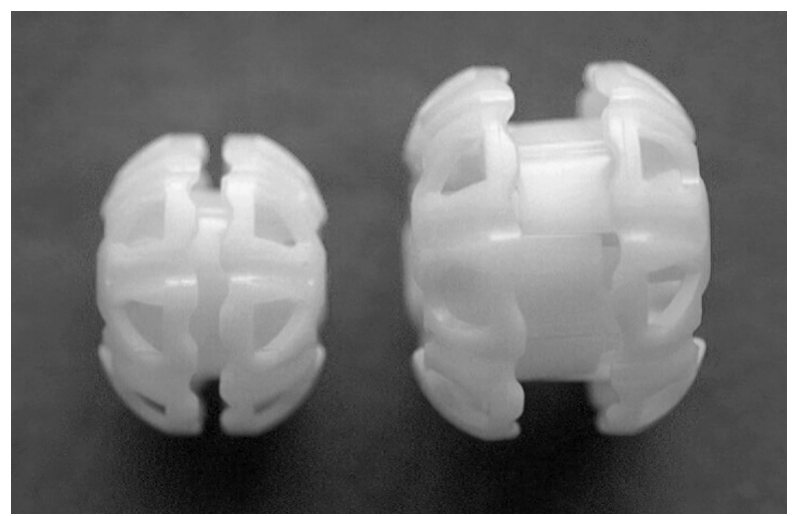

Fig. 1. The Biofragmentable Anastomosis Ring Valtrac in opened and closed position. In closed position the device creates an inverting type of anastomosis with minimal marginal necrosis 
In the final closed position between the margins of the headpieces remains a gap that will approximate the bowel ends without causing necrosis. The two lateral segments of the BAR interdigit and in the opened position leave a $6 \mathrm{~mm}$ wide gap which in a fully closed position will remain $1.5-2-2.5 \mathrm{~mm}$. This allows an adequate blood supply at the anastomotic site. The ring is supplied in 25,28 , $31,34 \mathrm{~mm}$ external diameter sizes. The rings can be chosen in different sizes according to the bowel lumen diameter and wall thickness (Zederfeldt et al., 1990).

Thirty mongrel dogs with an average weight of $14.9 \mathrm{~kg}$ (range: 9-24 kg), kept in separate cages, were used in the study. They were deprived of food $12 \mathrm{~h} \mathrm{be-}$ fore the intervention. Surgery was performed in general anaesthesia with endotracheal intubation (6.5-7.5 Rüsch tubes) and assisted respiration with halothane/oxygen with the following medication: premedication - Acepromazine 0.1 $\mathrm{mg} / \mathrm{kg}$, Methadone $1 \mathrm{mg} / \mathrm{kg}$, Atropine $0.05 \mathrm{mg} / \mathrm{kg}$; induction - Propophol $4 \mathrm{mg} / \mathrm{kg}$. They were monitored during the operation with an Ohmeda type pulse-oxymeter and they received isotonic glucose infusion via a cephalic vein catheter. They received a one-shot prophylactic antibiotic therapy (Cephamandole $0.5 \mathrm{~g}$ ).

\section{Surgical procedure}

We performed a left lateral thoracotomy in the sixth intercostal space. After isolation of the thoracic oesophagus and identification of the descending aorta, vagal nerve and pulmonary vessels, we transected the oesophagus above (proximal site) or under (distal site) the tracheal bifurcation and performed an end-to-end anastomosis between the oesophageal stumps. BAR anastomotic technique: we inserted a purse-string suture in both oesophageal ends with an absorbable monofilament thread composed of polyglycolic acid (Maxon 3/0), then the ring was introduced first in one of the oesophageal ends and the purse-string tied, and then the same manoeuvre was performed for the other stump. After the oesophageal ends were fixed on the two mushroom-shaped headpieces of the BAR, we checked if the oesophageal stumps were not rotated and then the ring was closed with bimanual, symmetric pressure. Care should be taken not to apply too hard compression, to avoid damage to the oesophageal wall grasped between our fingers and the ring. Ring sizes where chosen according to the diameter of the oesophageal lumen and the thickness of oesophageal wall (most suitable rings were those of 25 or $28 \mathrm{~mm}$ diameter and 2 or $2.5 \mathrm{~mm}$ gap size). The thoracic wall was closed with running sutures in three layers after desufflation.

Each dog received a tube-gastrostomy (Foley catheter) through a small left paracostal laparotomy for postoperative enteral feeding, started on the 2nd day with semi-liquid concentration diet (Waltham) and maintained for 7 days after surgery.

Animals were divided into two groups according to the site of the anastomosis and into subgroups according to the date of in vivo endoscopy and autopsy examination (Table 1). 
Table 1

Number of animals according to autopsy date and localisation of the anastomosis

\begin{tabular}{lccc}
\hline \multirow{2}{*}{ Autopsy day } & \multicolumn{3}{c}{ BAR } \\
\cline { 2 - 4 } & Proximal & Distal & Total \\
\hline 4th & 6 & 4 & 10 \\
7 th & 3 & 2 & 5 \\
14 th & 3 & 2 & 5 \\
28 th & 4 & 4 & 8 \\
\hline Total & 16 & 12 & 28 \\
\hline
\end{tabular}

proximal - above the tracheal bifurcation; distal - under the tracheal bifurcation; autopsy day - time after the operation

In each case before euthanasia we performed in vivo oesophagoscopy in general anaesthesia. After endoscopy the animals were killed by intracardial injection of saturated $\mathrm{KCl}$ solution.

At autopsy the thoracic cavity was opened and we performed a macroscopic gross examination of the anastomosis left in place, then the oesophageal segment containing the anastomosis was removed, opened longitudinally and the ring taken out. After macroscopic examination of the internal anastomotic surface, samples for histology were taken.

In vivo video-endoscopy was performed with an Olympus GIF-D4 type endoscope. We looked for mucosal lesions (bleeding, haematoma, erosions, ulceration, granulation tissue) close to the anastomotic site and ring. We also searched for compressive ischaemic lesions of the oesophageal wall due to the intraluminal presence of the ring or lesions related to the tracheal cartilages.

We performed a total of 30 BAR oesophageal anastomoses. Out of this we had two postoperative deaths not related to the surgical procedure (lung infection), therefore these cases were excluded from the study. Statistical calculations (Fisher's exact test) were performed by the Solo 3.1 version statistical package.

\section{Results}

Postoperative mortality rate was $13.3 \%$ (4 dogs out of 30 operated) with two deaths not related to the surgical procedure (excluded). We had 4 leaks out of 28 anastomoses (overall leakage rate: 14.3\%), of which two were subclinical in the 4th day subgroup, discovered only at autopsy examination. One anastomosis was located proximal, the other distal to the tracheal bifurcation. The other two leaks were: one in the 7th day group caused by accidental feeding with solid 
food and the other in the 14th day subgroup with a clinically relevant leakage. Therefore we could not perform endoscopy in 2 out of the 28 dogs.

Oesophagoscopy practised in general anaesthesia was easy to perform. We inflated only small amounts of air because the oesophagus close to the anastomosis was kept opened by the ring itself and there was no need for much air to have the oesophageal lumen in opened position. The rings were still in place in all cases on the 4th, 7th and 14th days but were all eliminated in the 28th day subgroup. In the 14th day subgroup in two cases the fragmentation of the ring was started but not completed. The other two rings seemed to be of the same shape as on the first days. We did not observe any difference in the endoscopic view of proximal or distal anastomoses. In the proximal group, where the anastomosis was situated in the paratracheal region in the vicinity of the tracheal cartilages we could not see any external compression or ischaemic lesions of the oesophageal wall that could be caused by the tracheal cartilages. The most frequent mucosal changes found at endoscopy in comparison with lesions detected at autopsy are presented in Table 2.

Table 2

Comparison of mucosal lesions found at endoscopy and autopsy examination

\begin{tabular}{|c|c|c|c|c|c|c|c|c|c|c|c|}
\hline \multirow{2}{*}{ Lesions } & \multicolumn{2}{|c|}{4 th } & \multicolumn{2}{|c|}{ 7th } & \multicolumn{2}{|c|}{14 th } & \multicolumn{2}{|c|}{ 28th } & \multirow{2}{*}{$\begin{array}{l}\mathrm{E} \\
\text { total }\end{array}$} & \multirow{2}{*}{$\begin{array}{l}\mathrm{A} \\
\text { total }\end{array}$} & \multirow{2}{*}{$\begin{array}{c}\text { Sensitivity } \\
\%\end{array}$} \\
\hline & $\mathrm{E}$ & A & $\mathrm{E}$ & A & E & A & $\mathrm{E}$ & A & & & \\
\hline Bleeding & 3 & 0 & 0 & 0 & 0 & 0 & 0 & 0 & 3 & 0 & \\
\hline Haematoma & 4 & 6 & 1 & 2 & 0 & 0 & 0 & 0 & 5 & 8 & 62.50 \\
\hline Erosion & 3 & 5 & 1 & 2 & 0 & 0 & 0 & 0 & 4 & 7 & 57.10 \\
\hline Ulceration & 3 & 3 & 2 & 2 & 0 & 0 & 0 & 0 & 5 & 5 & \\
\hline Slight granulation & 4 & 6 & 3 & 5 & 2 & 2 & 0 & 0 & 9 & 13 & 69.20 \\
\hline Severe granulation & 4 & 4 & 0 & 0 & 0 & 0 & 0 & 0 & 4 & 4 & \\
\hline Leaks & 0 & 2 & 0 & 1 & 0 & 1 & 0 & 0 & 0 & 4 & \\
\hline Total number of cases & 10 & 10 & 4 & 5 & 4 & 5 & 8 & 8 & 26 & 28 & \\
\hline
\end{tabular}

E: Number of lesions found at endoscopy; A: Number of lesions founded at autopsy; E total: Total number of endoscopic lesions; A total: Total number of lesions found at autopsy

Endoscopy detected the most frequent lesions in the 4th and 7th day group. Thirty-five percent of cases from these two subgroups presented small submucosal haematomas situated in the area that was in contact with the headpiece of the ring. Erosions and ulcerations (mucosal lesions of 2-4 mm diameter covered by fibrin deposits) were found in $64 \%$ of cases from the first postoperative week. Between the lateral holes of the BAR and in the anastomotic line we could detect during the first two weeks slight or pronounced granulation tissue in $72 \%$ of the examined cases (Figs 2 and 3). 

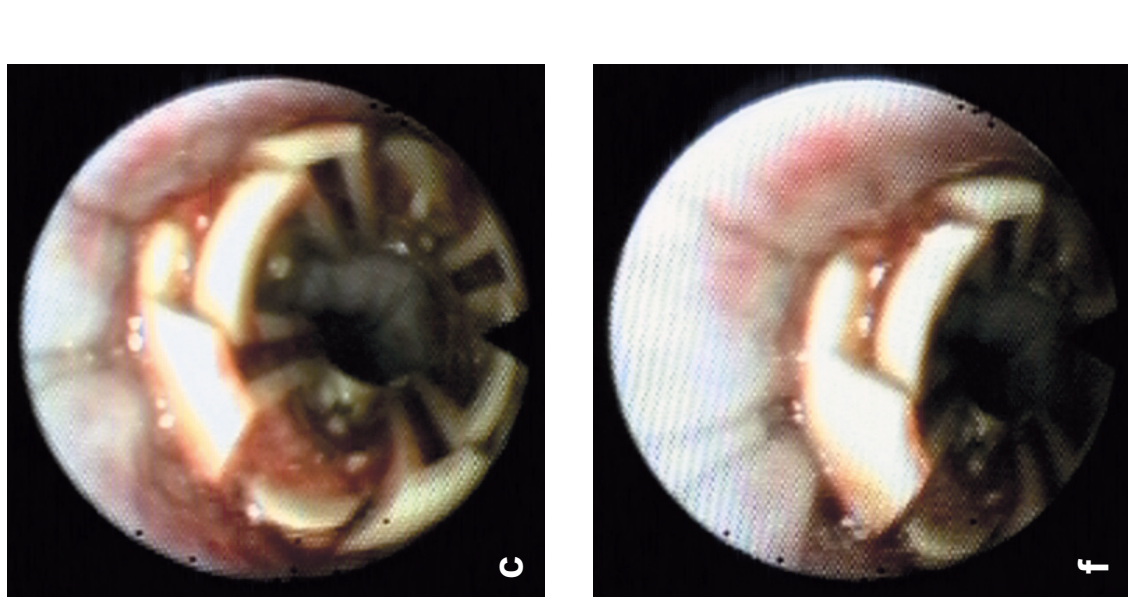

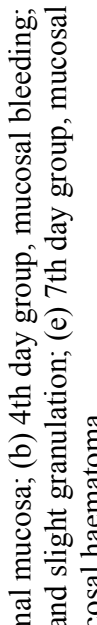
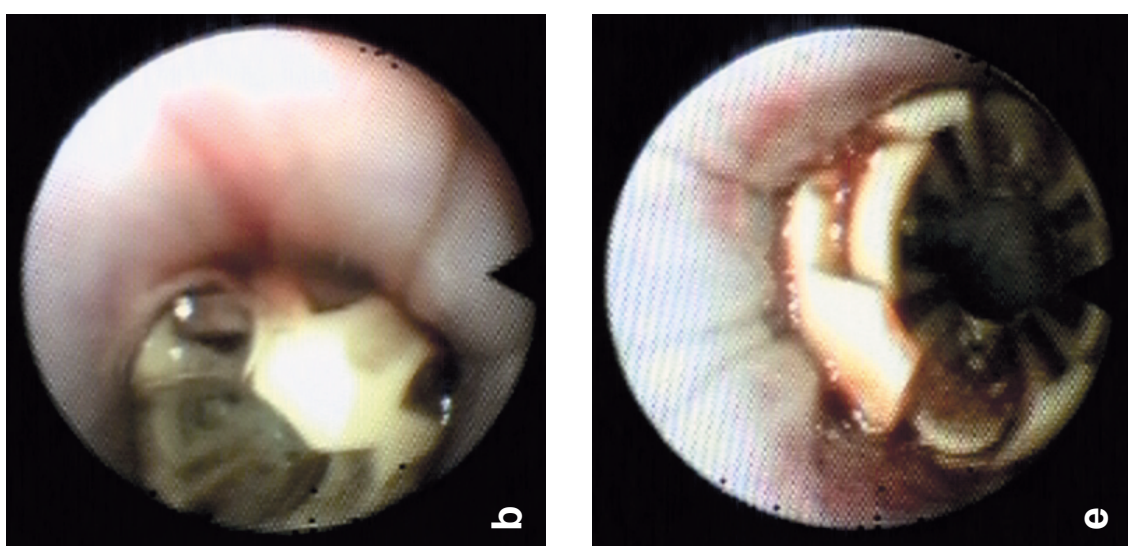

흥. 클

今ิ

फ़ 畓

密记

辛言吉

๘

氙 突

可

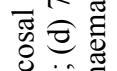

吾. 总

()
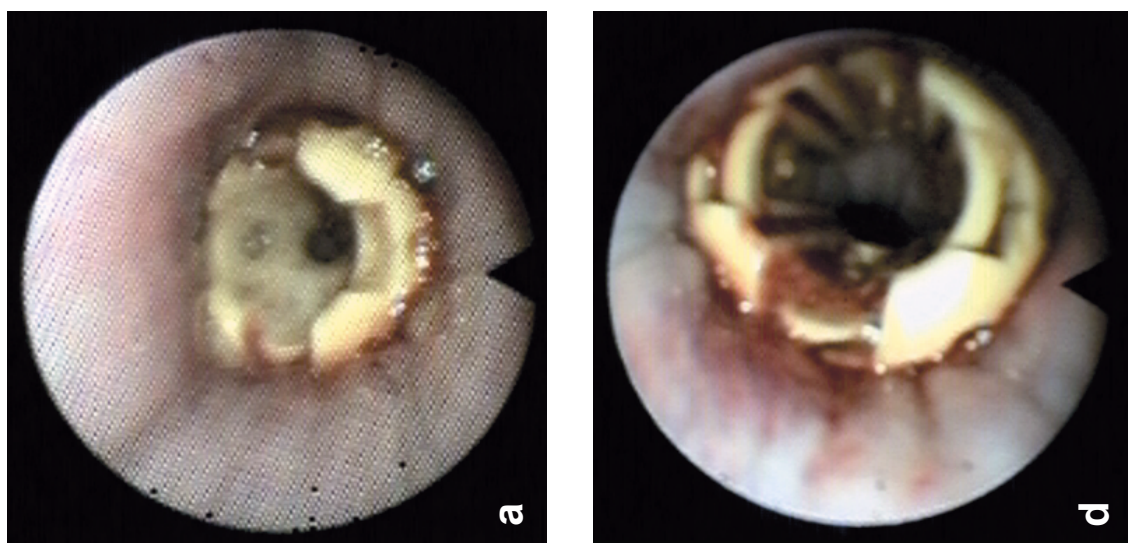

究

鸮

ส

还 完

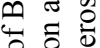

弪

$\frac{9}{5}$

. ज

:0

응

今े

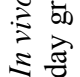

就导 

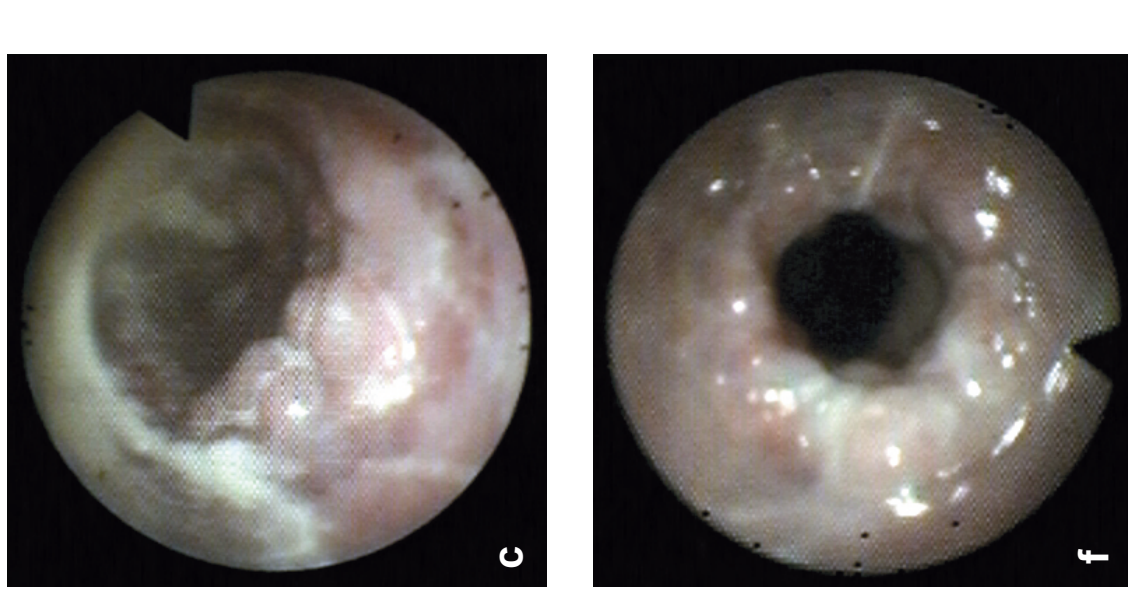

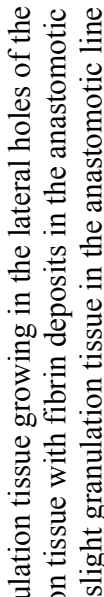
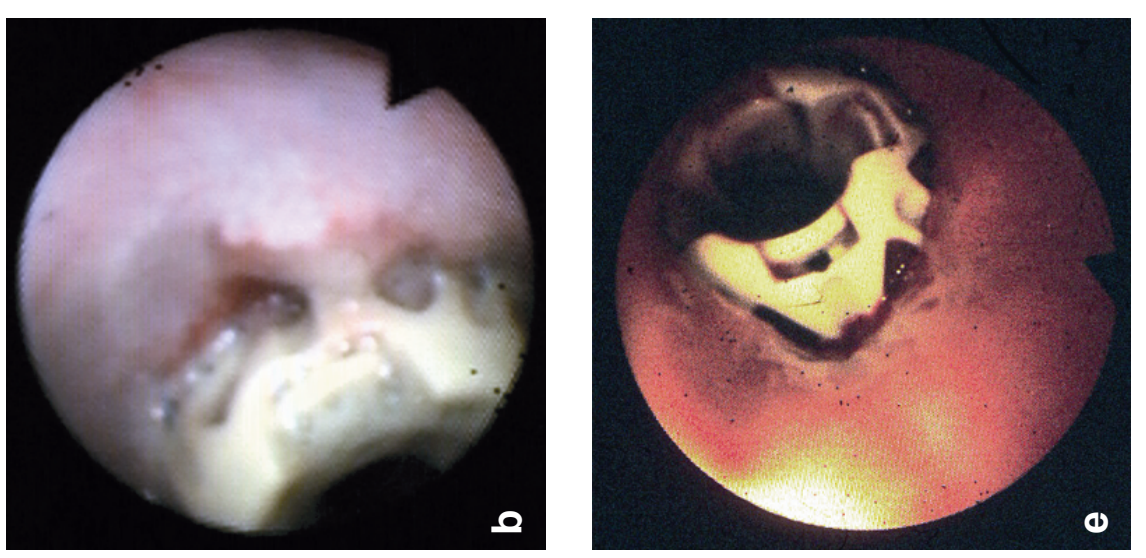

营壱

ڤ․ㅠ

흔 है तो

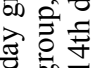

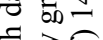

突可

త.

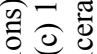

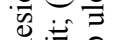

范

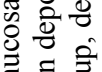
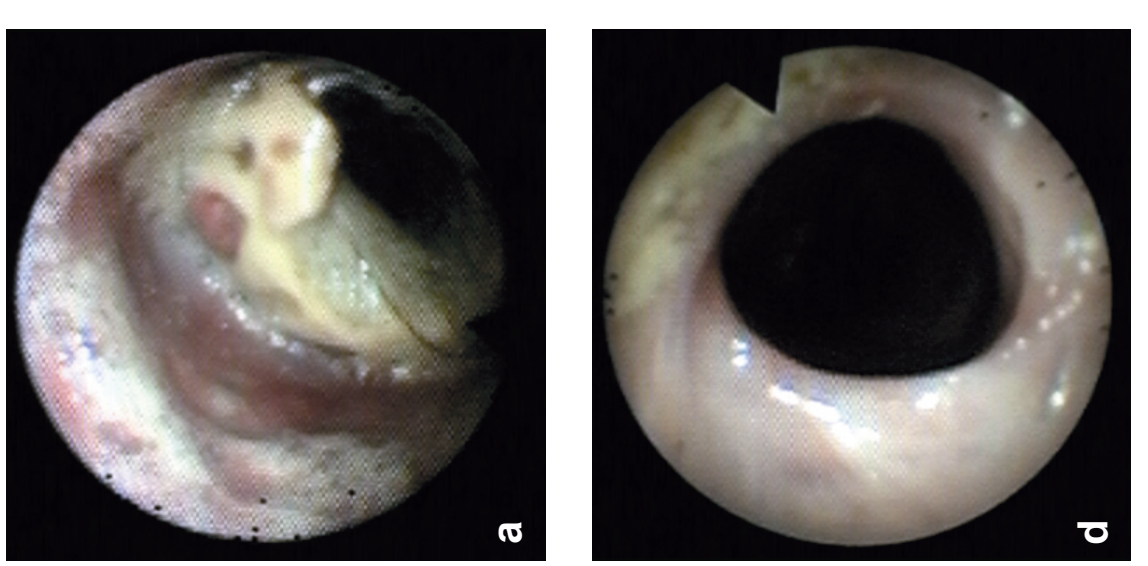

究

䠉吉

告

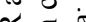

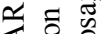

苓馿苛

立要要

可

$\therefore$

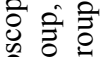

o to 50

可

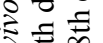

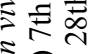

드웡

这自骂 
In the two cases with subclinical leak we could not see at endoscopy any fistula, but only ulcerations and a pronounced granulation tissue covered by fibrin deposits.

In the 14th day group endoscopy found a completely changed aspect. The ring was still in place but the mucosa adjacent to the ring was smoother, without pronounced inflammation and with a mild granulation tissue in two cases out of four. In the 28th day group the rings had already been eliminated, the site of the anastomosis was observed as a sharp circular edge without any mucosal lesions.

At autopsy we performed a macroscopic gross examination of the oesophageal anastomosis left in situ. After opening the pleural cavity we searched for leaks and adhesions between the anastomosis and thoracic organs. The lesions observed are presented in Table 3.

From the 4th postoperative day anastomoses were covered by pleural adhesions and fibrin deposits. In two cases when we started to separate the anastomosis and the small adhesions were cut and the fibrin deposits taken off, we observed in the anastomotic line dehiscences of 3-5 mm length with marginal necrosis. These leaks were completely blocked and sealed by fibrin and adhesions. In the 14th day case with clinical leakage we found a dehiscence of $15 \mathrm{~mm}$ and the margins of the BAR could be seen from outside. In contact with the leak there was a perianastomotic abscess with marginal necrosis of the oesophageal wall. The abscess was blocked by pleural adhesions.

The most frequent adhesions found at the anastomotic site were with the visceral pleura $(92.8 \%)$ and descending aorta $(67.8 \%)$. In case of proximal anastomoses we also found tracheal adhesions in $62.5 \%$ of cases. There was no significant difference regarding adhesions between the anastomosis and different mediastinal organs according to the localisation of the BAR (proximal or distal). The only difference was that in the proximal group we found tracheal adhesions too (in 10 dogs out of 16), due to the paratracheal localisation.

At the end the oesophageal segment containing the anastomosis was removed and opened longitudinally. The internal surface of the anastomotic site was examined and the changes (Fig. 4) found were compared with the endoscopic lesions as shown in Table 2.

There was no significant difference between the number of lesions found at in vivo endoscopy and at macroscopic examination during autopsy, although the overall sensitivity of endoscopy to discover mucosal lesions as compared to the autopsy findings was $73.1 \%$. Severe granulation and ulceration of the mucosa adjacent to the headpiece of the ring were recognised in all cases but more superficial, mild lesions (erosion, slight granulation, haematoma) were sometimes missed by endoscopy. 
Table 3

Macroscopic gross examination of BAR anastomoses

\begin{tabular}{lrrr}
\hline \multirow{2}{*}{ Lesions } & \multicolumn{2}{c}{ BAR no. (\%) } & \multirow{2}{*}{ Total BAR no. (\%) } \\
\cline { 2 - 3 } & Proximal & Distal & \\
\hline Leaks & $2(12.5)$ & $2(16.6)$ & $4(14.28)$ \\
Tracheal adhesions & $10(62.5)$ & 0 & $10(35.7)$ \\
Lung adhesions & $14(87.5)$ & $12(100)$ & $26(92.8)$ \\
Aortic adhesions & $9(56.2)$ & $10(83.3)$ & $19(67.8)$ \\
\hline Total number of anastomoses & 16 & 12 & 28 \\
\hline
\end{tabular}

\section{Discussion}

Due to anatomical and physiological features (absence of serosal layer, longitudinal muscle fibre distribution, segmental vascularisation) of the oesophagus, anastomotic leaks occur frequently (Dietz et al., 1995). Even with a correct surgical technique the leakage rate is between $14-35 \%$ in humans (Lee et al., 1994). Conventionally intrathoracic oesophageal anastomoses are hand-sewn or stapled (EEA circular stapler). The BAR has been used mainly in colonic surgery and also for anastomoses in the upper abdominal gastrointestinal tract with good results (overall leakage rate of $2 \%$ reported by Thiede et al., 1998) comparable to the conventional techniques even in 'high risk' conditions such as irradiated bowel (Croston et al., 1990), steroid treatment (Maney et al., 1988) and emergency surgery as well (Di Castro et al., 1998). As previous reports showed, the BAR anastomoses have a high mechanical stability in the first postoperative days, with the highest bursting strength if compared to stapled or hand-sewn anastomoses (Zederfeldt et al., 1990).

Because of the gap-width of the ring the anastomosis is created only by atraumatic, gentle pressure exercised on the oesophageal wall, without creating large tissue necrosis (Cossu et al., 2000). The ring is fragmented and eliminated from the oesophagus between 14 and 21 days, leaving no foreign material at the anastomotic site, therefore the risk of stenosis is reduced. The procedure is time saving, it has a standard, uniform technique all over the gastrointestinal tract and has no higher postoperative complication rate than the conventional procedures (Maney et al., 1988).

We studied the feasibility of the BAR for intrathoracic oesophageal surgery and examined the healing of the anastomoses performed in different localisations. We also checked the usefulness and sensitivity of the endoscopy to recognise early postoperative signs of compromised or abnormal healing of the anastomosis. 
KOVÁCS et al.

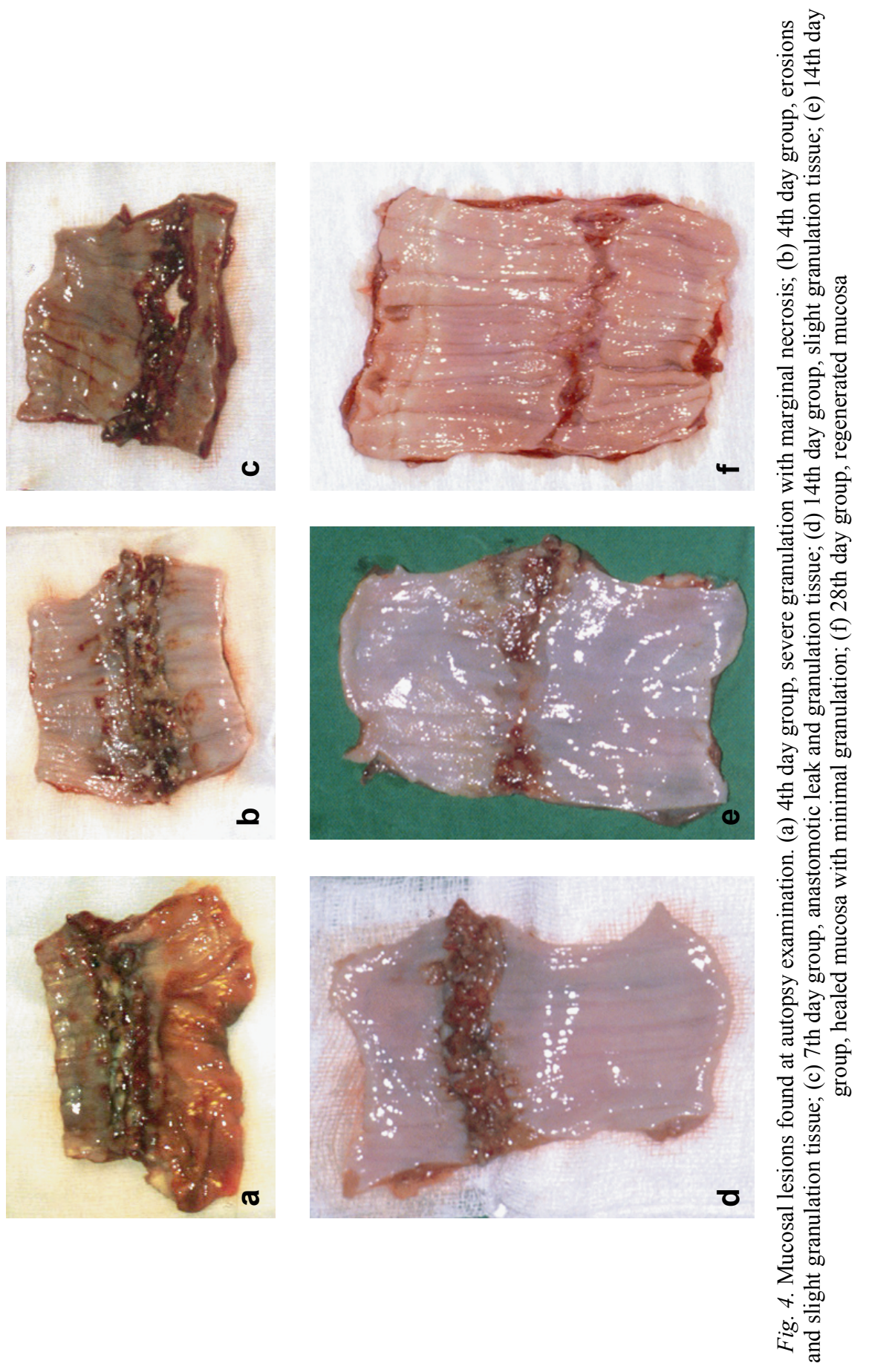


There was no technical difficulty to perform end-to-end anastomoses with the BAR either on the paratracheal or on the distal oesophagus in dogs. It is necessary to use a ring with a suitable diameter and gap-size for a safe and easy anastomosis. The BAR maintained the oesophageal stumps in inverted position until the healing was completed (the rings were still in place in the 14th day group). The large central hole in the middle of the ring permits an early passage for fluids and semifluids. The high mechanical strength during the first week allows to use the procedure in 'difficult' conditions (e.g. inflammation).

We did not find postoperative complications (leaks) related to the localisation of the anastomosis. The vicinity of the trachea did not cause external compressive lesions of the oesophageal wall at the site of the anastomosis. Tracheal adhesions did not develop later in fistulas. In the posterior mediastinum of dogs there is enough place for the oesophagus anastomosed with the BAR. Generally adhesions may help to heal small, subclinical fistulas.

Oesophagoscopic follow-up of intrathoracic oesophageal BAR anastomoses has not been previously reported.

In our experimental model we studied the applicability of oesophagoscopy for the follow-up of oesophageal anastomoses performed with the BAR and compared the endoscopic findings to lesions observed at autopsy examination.

Due to the special construction of the BAR (mushroom-shaped headpieces with a central lumen), the oesophagus is maintained open as soon as the anastomosis is finished (Fig. 5).

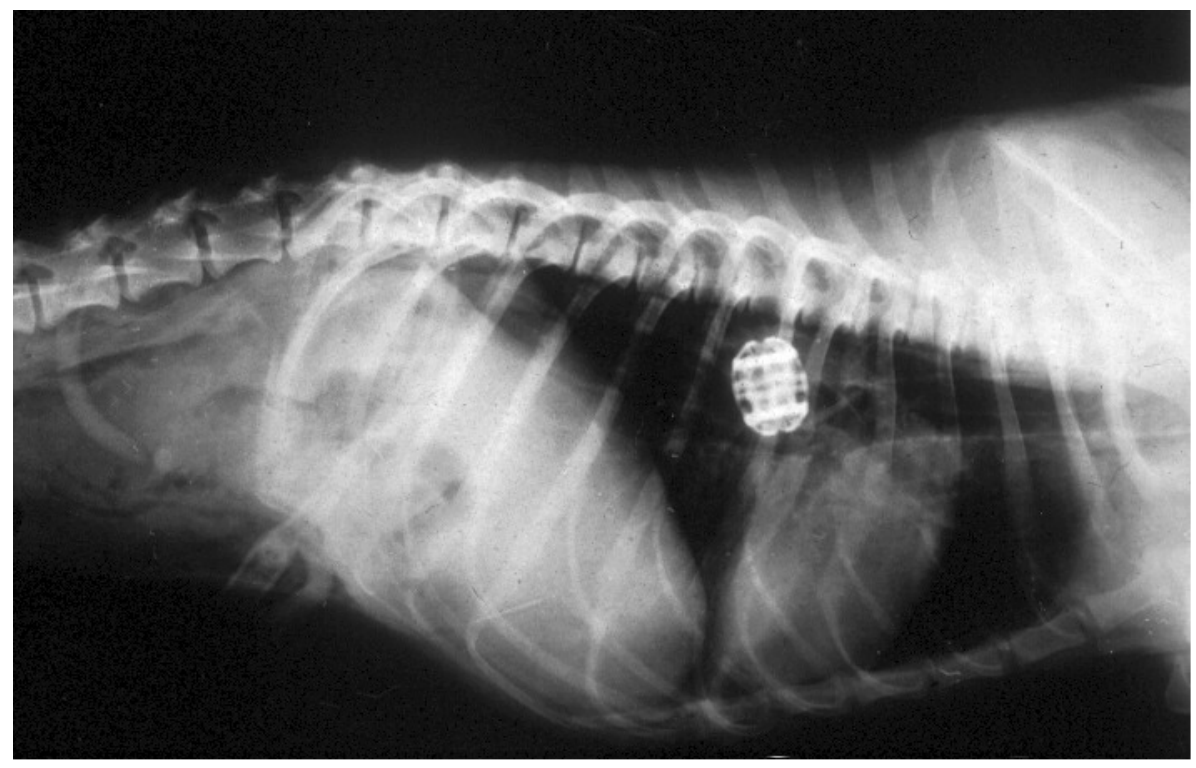

Fig. 5. Radiological view (chest X-ray) of a BAR anastomosis situated in the vicinity of the trachea (7th day group) 
The ring will be eliminated only after the second week when the anastomosis has already healed, as proved by our results. The opened oesophageal lumen permits an easy endoscopic examination with small quantities of air insufflation, therefore there is no risk of damage to the anastomosis in the early postoperative days.

The two small subclinical leakages were missed by endoscopy. At autopsy the leaks were covered by adhesions and fibrin deposits. Probably they would have healed without any problem due to the sealing effect of pleural adhesions. At endoscopy we could only see a pronounced granulation tissue and ulcerations on the oesophageal wall that was in contact with the surface of the BAR headpiece.

Endoscopy was suitable to detect bleedings in the 4th day subgroup. These bleedings from the submucosal layer may develop in submucosal haematomas which can compromise the healing of the anastomosis. Therefore it is important to make a careful haemostasis of small bleedings from the oesophageal edges before the purse-string suture is done. Mild mucosal lesions such as erosions and slight granulation tissue can be missed by oesophagoscopy. Larger lesions such as ulcerations (which may later develop in fistulae) and pronounced granulation tissue (characteristic of an inflammatory reaction in the anastomosis) were recognised in all cases.

In conclusion, oesophagoscopy is a feasible procedure with an overall sensitivity of $73.1 \%$ to detect mucosal lesions. Due to mechanical characteristics of the BAR (keeps the oesophageal lumen open, gives a high bursting strength during the first postoperative week), endoscopic visualisation of oesophageal BAR anastomoses is a simple and safe method of follow-up and may help predict the normal or compromised healing of the anastomosis.

\section{References}

Bundy, C. A., Zera, R. T., Onstad, G. A., Bilodeau, L. L. and Bubrick, M. P. (1992): Comparative surgical and colonoscopic appearance of colon anastomoses constructed with sutures, staples and the biofragmentable anastomotic ring. Surg. Endosc. 6, 18-22.

Cossu, M. L., Coppola, M., Fais, E., Ruggiu, M., Spartá, C., Profili, S., Bifulco, V., Meloni, G. B. and Noya, G. (2000): The use of the Valtrac ring in the upper and lower gastrointestinal tract for single, double and triple anastomoses: A report of 50 cases. Am. Surgeon. 66, $759-762$.

Croston, J. K., Jacobs, D. M., Kelly, P. H., Feeney, D. A., Johnston, G. R., Strom, R. L. and Bubrick, M. P. (1990): Experience with the Biofragmentable Anastomotic Ring (BAR) in bowel preoperatively irradiated with 6000 rad. Dis. Col. \& Rect. 33, 222-226.

Di Castro, A., Biancari, F., Brocato, R., Adami, E. A., Truosolo, B. and Massi, G. (1998): Intestinal anastomosis with the Biofragmentable Anastomosis Ring. Am. J. Surg. 176, 472-474.

Dietz, U. A. (1994): Eine experimentelle Vergleichsstudie zur Nahttechnik von ösophagoösophagealen Anastomoses im Halsbereich. Handnaht vs mechanische Naht-BAR. Inaugural Dissertation, Würzburg. 
Dietz, U. A., Czeczko, N. G., Polonio, B., Repka, J. C. D., Mulinari, A., Engemann, R. and Thiede, A. (1995): Hand-sewn anastomosis versus Biofragmentable Anastomosis Ring in the cervical esophagus of dogs: A comparative experimental study. In: Engemann, R. and Thiede, A. (eds) Compression Anastomosis by Biofragmentable Rings. Proceedings of the Second European Workshop. Springer Verlag, Berlin - Heidelberg. pp. 35-43.

Hardy, T. G., Aguilar, P. S., Stewart, W. R. C., Katz, A. R., Maney, J. W., Costanzo, J. T. and Pace, W. G. (1987): Initial clinical experience with the biofragmentable ring for sutureless bowel anastomosis. Dis. Col. \& Rect. 30, 55-61.

Kovács, T., Köves, I., Sulyok, Z. and Besznyák, I. (1996): Safe alternative to conventional anastomosis techniques. Initial experience with the biofragmentable anastomosis ring Valtrac. Br. J. Surg. 83 (Suppl. 2), 39.

Kovács, T., Köves, I., Vámosi, N. I. and Németh, T. (1998): Biofragmentable anastomosis ring used for esophageal anastomosis: experimental and early clinical results. Eur. J. Surg. Oncol. 24, 252 .

Lee, Y., Fujita, H., Yamana, H. and Kakegawa, T. (1994): Factors affecting leakage following esophageal anastomosis. Surg. Today 24, 24-29.

Maney, J. W., Katz, A. R., Lehmann, K. L., Pace, W. G. and Hardy, T. G. (1988): Biofragmentable bowel anastomosis ring: Comparative efficacy studies in dogs. Surgery 103, 56-62.

Thiede, A., Geiger, D., Dietz, U. A., Debus, E. S., Engemann, R., Lexer, G. C., Lunstedt, B. and Mokros, W. (1998): Overview on compression anastomoses: Biofragmentable Anastomosis Ring multicenter prospective trial of 1666 anastomoses. World. J. Surg. 22, 78-87.

Zederfeldt, B., Jiborn, H. and Ekelund, G. (1990): Sutureless colonic anastomoses. Langenbecks Arch. Chirurgie 375, 181-185. 